\title{
Numerical Vibration Displacement Solutions of Fractional Drawing Self-Excited Vibration Model Based on Fractional Legendre Functions
}

\author{
Jiaquan Xie $\mathbb{D},{ }^{1}$ Yongjiang Zheng, ${ }^{2}$ Zhongkai Ren, ${ }^{1}$ Tao Wang $\mathbb{D},{ }^{1}$ and Guangxian Shen ${ }^{2}$ \\ ${ }^{1}$ College of Mechanical and Vehicle Engineering, Taiyuan University of Technology, Taiyuan 030024, Shanxi, China \\ ${ }^{2}$ School of Mechanical Engineering, Yanshan University, Qinhuangdao 066004, Hebei, China \\ Correspondence should be addressed to Tao Wang; 2178263018@qq.com
}

Received 22 April 2019; Accepted 26 September 2019; Published 6 December 2019

Academic Editor: Qingling Wang

Copyright (c) 2019 Jiaquan Xie et al. This is an open access article distributed under the Creative Commons Attribution License, which permits unrestricted use, distribution, and reproduction in any medium, provided the original work is properly cited.

In practice, due to the fact that the phenomenon of drawing self-excited vibration can be deemed as one of the hunting phenomena of the mechanical system, this study focuses on investigating the drawing self-excited vibration process through proposing the fractional differential equation model of hunting phenomenon of the mechanical system. The fractional Legendre functions together with their fractional differential operational matrices are used to numerically solve the model. In this way, the numerical solutions of vibration displacement of the model are obtained. At the end, the proposed model and algorithm are proved to be effective via analyzing the numerical results and phase position.

\section{Introduction}

The self-excitation vibration exists in the natural and engineering field, which requires no external force or external action to change the structural parameters of the system but relies on the interaction of various components within the system to maintain the steady-state periodic motion [1-8]. Some self-excited oscillations are very complicated. For example, the human circulatory system is an extremely complex self-excited vibration system. The heart vibrates at a certain frequency and intensity to maintain blood flow in the blood vessels, which is a typical self-excited vibration phenomenon.

The hunting phenomenon of the mechanical system exists in a low-speed drive system with strong friction force and frequently occurs in unstable and unevenly stopped motion, which sometimes is called as stick-slip, or slip. The hunting phenomenon is the main reason for the self-excited vibration of the mechanical system. Once the hunting phenomenon occurs, the mechanical transmission becomes unstable so as to be impossible to achieve accurate measurement and precision machining. To avoid the hunting phenomenon, the theory analysis and experiment research should be carried out. In order to obtain quantitative results, a simplified mechanical model with a single degree of freedom and a quantitative description of the friction force should be established.

The steel strand drawing technology has been investigated for hundreds of years [9]. In the 12th century, there were blacksmiths and drawers. In the middle of the 13th century, Germany first produced the hydraulic drawing machine to promote in the world, which ushered in a new era of mechanization. For drawing machine with wire processing, when the surface reduction rate, drawing speed, and lubrication parameter are improper and exceed a reasonable range, there will be a hunting or jumping phenomenon. For example, the surface quality of the wire is not smooth, and the defect leads to the flutter accident of the puller.

Hao et al. [10] carried out an in-depth theoretical analysis on the mechanism and conditions of chattering grains generated in the process of cold drawing of tapered short mandrel steel tubes. Yang [11] used the finite element method to analyze the vibration and self-excited vibration of 
the four-high rolling mill. Lu et al. [12] studied the phenomenon of self-excited vibration of $1420 \mathrm{~mm}$ cold mill, established the mathematical model of the roll and solved it with the method of multiscale perturbation, and proposed a method to solve the self-excited vibration. Erdbrink and Krzhizhanovskaya [13] used the differential evolution method to analyze the second-order time signal of the selfexcited vibration equation. Aarsnes and Aamo [14] predicted the occurrence of self-excited vibrations during drilling using an infinite dimensional model. Zhang et al. [15] applied a nonlinear friction work formulation for the analysis of self-excited vibrations.

Recently, fractional partial differential equations have been widely applied in various fields of science and engineering [16-18], and wave equation is one of the most important models of them. Over the past decade, the modelling and control of several classes of wave process have been researched a hot point $[19,20]$. In [21], Jiang et al. analyzed the boundary control strategy for a fractional wave equation with external disturbances. In [22], the numerical approximation of the time fractional diffusion-wave equation in a semi-infinite channel by using the finite difference method and Laguerre-Legendre spectral method is carried out. In [23], time fractional diffusion-wave equation with damping has been solved by using the method of separation of variables.

In this study, in order to more accurately describe the whole process of drawing self-excited vibration, a fractional differential equation model is proposed, and the fractionalorder Legendre functions are utilized to obtain the numerical vibration displacement solutions.

\section{Fractional Dynamic Model of Mechanical System Hunting Phenomenon}

Although some studies considered mechanical system hunting phenomenon [24], to our best knowledge, the fractional-order dynamic model to characterize hunting phenomenon of mechanical system is first presented, as shown in Figure 1. The diagram of drawing plastic working [25] is depicted in Figure 2. Figure 2 shows the deformation of drawing long parts from coarse to fine in the drawing process.

The fractional differential equation of mechanical system hunting phenomenon is given as follows:

$$
m \ddot{u}+c\left(D_{t}^{\alpha} u-D_{t}^{\alpha} u_{0}\right)-\varphi(\dot{u})+k\left(u-u_{0}\right)=0, \quad 0<\alpha \leq 1,
$$

where $D_{t}^{\alpha}$ denotes the fractional differential operator defined in the Caputo sense [26,27], $m$ is the quality of the slider, $c$ is the fractional damping coefficient, $k$ is the spring stiffness coefficient, $u_{0}$ is the displacement at drive end, $u$ is the slider displacement, and $\varphi(\dot{u})$ is the frictional force.

If the slider is driven at constant speed, setting the speed of the driving end as $v$, then the displacement $u_{0}$ of the driving end $T$ can be expressed as

$$
u_{0}=v t
$$

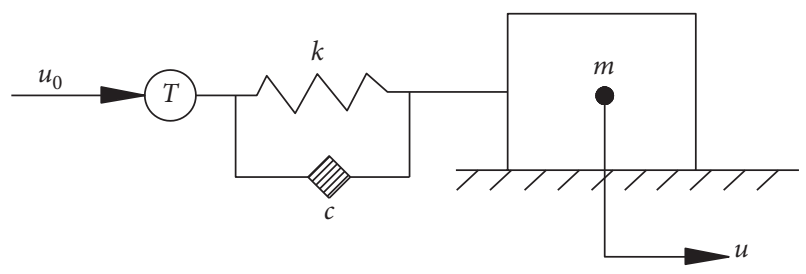

FIgURE 1: The diagram of fractional dynamic model of mechanical system hunting phenomenon.

Substituting equation (2) into Equation (1), we get

$$
m \ddot{u}+c\left(D_{t}^{\alpha} u-v D_{t}^{\alpha} t\right)-\varphi(\dot{u})+k\left(u-u_{0}\right)=0 .
$$

Converting the slider displacement $u$ into the relation of relative displacement $x_{1}$ as

$$
u=x_{1}+u_{0}=x_{1}+v t
$$

then we get

$$
m \ddot{x}_{1}+c D_{t}^{\alpha} x_{1}+k x_{1}-\varphi\left(\dot{x}_{1}+v\right)=0 .
$$

Introducing the dimensionless time $\tau$ and defining the dimensionless displacement $x$ and damping ratio $\xi$ :

$$
\begin{aligned}
& \tau=\omega_{0} t\left(\omega_{0}=\sqrt{\frac{k}{m}}\right), \\
& x=\frac{\omega_{0} x_{1}}{v}, \\
& \xi=\frac{c}{2 \sqrt{k m}},
\end{aligned}
$$

the sliding block motion equation can be simplified as

$$
x^{\prime \prime}+2 \xi D^{\alpha} x+x-\frac{\varphi\left[\left(x^{\prime}+1\right) v\right]}{m \omega_{0} v}=0,
$$

where $x^{\prime}, x^{\prime \prime}$, and $D^{\alpha} x$ are the first, second, and $\alpha$ derivatives of $x$ with respect to $\tau$.

The Karnopp friction model is employed to describe friction $\varphi\left[\left(x^{\prime}+1\right) v\right]$. The difference between static and dynamic friction is

$$
\Delta F=F_{s}-F_{k}=\Delta \mu N\left(\Delta \mu=\mu_{s}-\mu_{k}\right) .
$$

The dimensionless dynamic friction coefficient $f$ and dimensionless static-dynamic friction drop $d$ are defined as follows:

$$
\begin{aligned}
& f=\frac{\mu_{k} N}{m \omega_{0} v}, \\
& d=\frac{\Delta \mu N}{m \omega_{0} v} .
\end{aligned}
$$

The sliding block motion equation is simplified again as

$$
x^{\prime \prime}+2 \xi D^{\alpha} x+x=-f \operatorname{sgn}\left(x^{\prime}+1\right)
$$




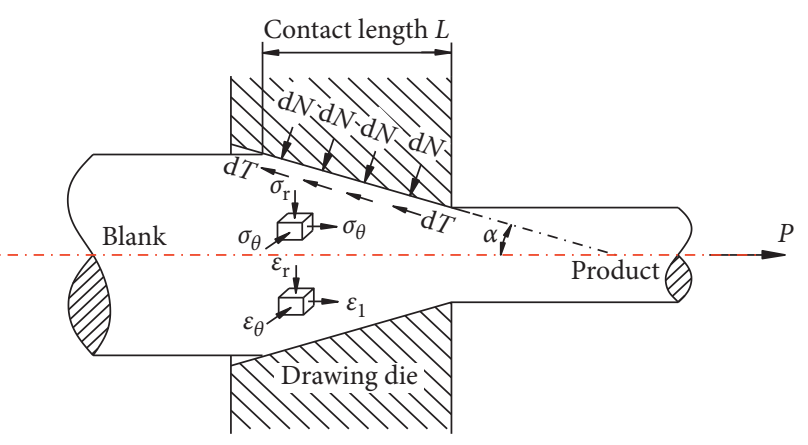

(a)

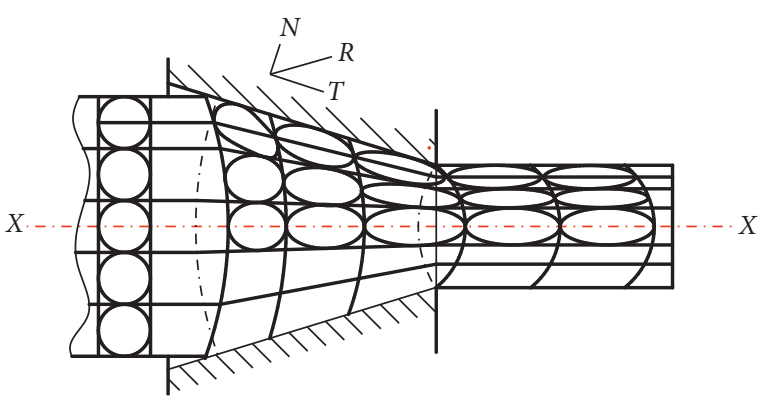

(b)

FIgURE 2: The diagram of drawing plastic working.

where $\operatorname{sgn}(x)$ is the sign function, namely,

$$
\operatorname{sgn}(x)=\left\{\begin{array}{cc}
1, & x>0, \\
0, & x=0, \\
-1, & x<0 .
\end{array}\right.
$$

Segmentation analysis:

(a) Sliding section before rolling:

$$
x^{\prime \prime}+2 \xi D^{\alpha} x+x=f, \quad 0<\alpha \leq 1 .
$$

(b) Sliding section after rolling:

$$
x^{\prime \prime}+2 \xi D^{\alpha} x+x=-f, \quad 0<\alpha \leq 1 .
$$

From equations (12) and (13), the general form of fractional differential equation model of mechanical system hunting phenomenon is established:

$$
y^{\prime \prime}(x)+2 \xi D_{x}^{\alpha} y(x)+y(x)=f(x), \quad 0<\alpha \leq 1,
$$

where $\xi$ are constants. With the initial conditions,

$$
\begin{aligned}
y(0) & =\delta_{1}, \\
y^{\prime}(0) & =\delta_{2} .
\end{aligned}
$$

\section{Computing Method}

3.1. Definitions. The generalized fractional-order Legendre functions (GFLFs), devoted by $F l_{i}^{h \alpha}(t)$, is defined by introducing the change of variable $t=x h$. Then, the GFLFs have recurrence formula as follows [28]:

$$
\begin{aligned}
F l_{i+1}^{h \alpha}(t)= & \frac{(2 i+1)\left(2(t / h)^{\alpha}-1\right)}{i+1} F l_{i}^{h \alpha}(t) \\
& -\frac{i}{i+1} F l_{i-1}^{h \alpha}(t), \quad i=1,2, \ldots,
\end{aligned}
$$

where $F l_{0}^{h \alpha}(t)=1$ and $F l_{1}^{h \alpha}(t)=2(t / h)^{\alpha}-1$.

The analytical form of the GFLFs $F l_{i}^{h \alpha}(x)$ of degree $i \alpha$ is given by

$$
F l_{i}^{h \alpha}(x)=\sum_{s=0}^{i} b_{s, i} \frac{t^{s \alpha}}{h^{s \alpha}}, \quad i=1,2, \ldots
$$

3.2. Functions Approximation. Suppose $y(x) \in L^{2}[0, h]$, it can be expanded in terms of the GFLFs as follows [29]:

$$
y(x)=\sum_{i=0}^{\infty} c_{i} F l_{i}^{h \alpha}(x)
$$

where $c_{i}$ are obtained by

$$
c_{i}=\frac{\alpha(2 i+1)}{h^{\alpha}} \int_{0}^{1} F l_{i}^{h \alpha}(x) f(x) \omega_{l}^{\alpha} \mathrm{d} x, \quad i=1,2, \ldots,
$$

where the weight function $\omega_{l}^{\alpha}(x)=x^{\alpha-1}$.

If we consider truncated series in equation (13), we obtain

$$
y(x) \approx y_{m}(x)=\sum_{i=0}^{m-1} c_{i} F l_{i}^{h x}(x)=\mathbf{C}^{T} \boldsymbol{\Phi}(x),
$$

where $\mathbf{C}=\left[c_{0}, c_{1}, \ldots, c_{m-1}\right]^{T}$ and $\boldsymbol{\Phi}(x)=\left[F l_{0}^{h \alpha}(x), F l_{1}^{h \alpha}(x)\right.$, $\left.\ldots, F l_{m-1}^{h \alpha}(x)\right]$.

3.3. The GFLF Fractional-Order Operational Matrix of Derivative. The derivative of the function vector $\Phi(x)$ can be approximated as follows:

$$
D^{\alpha} \Phi(x)=\mathbf{P}^{\alpha} \Phi(x),
$$

where $\mathbf{P}^{\alpha}$ is called the GFLF operational matrix of the derivative.

Theorem 1. Suppose $\mathbf{P}^{\alpha}$ is the $m \times m$ GFLF operational matrix of Caputo fractional derivatives of order $\alpha, \beta>\alpha / 2$, $\beta \notin \mathbb{N}$, then the elements of $\mathbf{P}^{\alpha}$ are given as follows [30]:

$$
\begin{aligned}
\left(p_{i j}\right)_{i, j=1}^{m-1, m-1}= & (2 j+1) \beta h^{-\alpha} \sum_{s=0}^{i} \sum_{r=0}^{j} b_{r, j} b_{s, i}^{\prime} \\
& \cdot \frac{\Gamma(s \beta+1)}{\Gamma(s \beta-\alpha+1)} \frac{1}{(s+r+1) \beta-\alpha},
\end{aligned}
$$


where $b_{s, i}^{\prime}=\left\{\begin{array}{cl}0, & s \beta \in N_{0} \text { and } s \beta<\alpha, \\ b_{s, i}^{\prime}=b_{s, i}, & s \beta \notin N_{0} \text { and } s \beta \geq \alpha \text { or } s \beta \in N_{0} \text { and } s \beta \geq \alpha .\end{array}\right.$

3.4. Solving Process. Substituting equations (18) and (21) into equation (14), we have

$$
\begin{aligned}
y^{\prime \prime}(x) & \approx \mathbf{C}^{T} \frac{d^{2} \Phi(x)}{d x^{2}} \approx \mathbf{C}^{T} \mathbf{P}^{2} \Phi(x), \\
D_{x}^{\alpha} y(x) & \approx \mathbf{C}^{T} \frac{d^{\alpha} \Phi(x)}{d x^{\alpha}} \approx \mathbf{C}^{T} \mathbf{P}^{\alpha} \Phi(x), \\
f(x) & \approx \mathbf{F}^{T} \boldsymbol{\Phi}(x)
\end{aligned}
$$

where the coefficient matrices $\mathbf{F}$ can be obtained by equation (19). Substituting equations (23)-(25) and (18) into equation (14), we get

$$
\mathbf{C}^{T} \mathbf{P}^{2} \boldsymbol{\Phi}(x)+2 \xi \mathbf{C}^{T} \mathbf{P}^{\alpha} \boldsymbol{\Phi}(x)+\mathbf{C}^{T} \boldsymbol{\Phi}(x)=\mathbf{F}^{T} \boldsymbol{\Phi}(x) .
$$

Simplifying equation (26), we obtain

$$
\mathbf{C}^{T} \mathbf{P}^{2}+2 \xi \mathbf{C}^{T} \mathbf{P}^{\alpha}+\mathbf{C}^{T}=\mathbf{F}^{T}
$$

For equation (15), we have

$$
\begin{aligned}
\mathbf{C}^{T} \boldsymbol{\Phi}(0) & =\delta_{1}, \\
\mathbf{C}^{T} \mathbf{P} \boldsymbol{\Phi}(0) & =\delta_{2} .
\end{aligned}
$$

Equation (27) together with equation (28) constitutes a system of linear algebraic equations. These unknown coefficients $c_{i}$ can be obtained by solving the system. A flowchart of the algorithm is given in Figure 3.

\section{Numerical Simulation}

In this section, several numerical experiments are presented to verify the established model and the provided algorithm. The parameters of the following examples are actual physical parameters. In the following numerical examples, $m$ represents the number of discrete terms of the polynomial; that is, the larger the value of $m$ is, the more discrete terms of the polynomial, the closer the numerical result approaches to the analytical result, but the corresponding calculation amount will also increase. In order to obtain effective numerical results and save calculation time, we take $m=4$.

Case 1. Considering the following fractional differential equations model of mechanical system hunting phenomenon with $\xi=0.1$ :

$$
\begin{aligned}
y^{\prime \prime}+2 \xi D^{0.5} y+y & =2+0.2 \times \frac{2}{\Gamma(2.5)} x^{1.5}+x^{2}, \\
y(0) & =y^{\prime}(0)=0 .
\end{aligned}
$$

The analytical solution of this problem is $y=x^{2}$. When $m=4$, the numerical and analytical solutions are shown in Figure 4 . In this case, the analytical result is given to verify the effectiveness of the proposed algorithm. Figure 4 shows that the proposed method can achieve a good agreement between numerical and analytical results.
Case 2. Considering the second-order wave equation with $\xi=0.1, f(x)=-1$. The analytical result of this problem is $y=e^{-\xi x}\left(c_{1} \cos \sqrt{1-\xi^{2}} x+c_{2} \sin \sqrt{1-\xi^{2}} x\right)-f$, here $c_{1}=c_{2}$ $=1, \xi=0.1$, and $f(x)=-1$. The numerical results with $m=$ 4, 5 and analytical results are shown in Figure 5. The main reason why the numerical solutions do not coincide the analytical solutions at the beginning is that the initial conditions are homogeneous in this example. However, when $m$ takes different values, the obtained numerical results are consistent, which proves that the proposed scheme is effective:

$$
y^{\prime \prime}+2 \xi y^{\prime}+y=f(x)
$$

Case 3. Considering the fractional-order wave equation with $f(x)=-1$ :

$$
y^{\prime \prime}+2 \xi D^{\alpha} y+y=f(x)
$$

When $m=4$ and $\alpha=0.5$, the numerical results with $\xi=$ $0.15,0.6$ are shown in Figure 6 . Figure 6 shows that the numerical results are frequency invariant attenuation waveforms. When $m=4$ and $\xi=0.15$, the numerical results with $\alpha=0.5,0.7,0.9$, and 1 are shown in Figure 7 . When $m=4$ and $\alpha=1$, the phase diagram with $\xi=0.15$ and $\xi=$ 0.6 is shown in Figure 8. Figure 8 shows that images with $\xi=0.6$ attenuate faster than those with $\xi=0.15$, which is consistent with the results in Figure 6. Figures 7, 10, and 13 show that the numerical vibration displacement solutions approach to the solution $(\alpha=1)$ with the fractional order $\alpha$ gradually approximating to 1 . These show that the fractional order model has memory and can extract short-term sequence details arbitrarily.

Case 4 (see [31]). Considering the fractional-order wave equation, in this case, we take the friction force $f=400$ $\left(1-e^{-20 x}\right)+100 \sin (6 \pi x)$. When $m=4$ and $\alpha=0.5$, the numerical results with $\xi=0.01,0.15$, and 0.6 are shown in Figure 9. Figure 9 shows that the vibration displacement solutions are still attenuated waveform, and the waveform is equiperiodic when $\xi=0.01$. Moreover, the waveforms no longer change regularly with $\xi$ increasing. When $m=4$ and $\xi=$ 0.15 , the numerical results with $\alpha=0.5,0.7,0.9$, and 1 are shown in Figure 10. When $m=4$ and $\alpha=1$, the phase diagram with $\xi=0.01, \xi=0.15$, and $\xi=0.6$ is shown in Figure 11 . Figure 11 shows that the waveform attenuation is the fastest when $\xi=0.6$. From Figures 6,9 , and 12, it can be concluded that the larger the value of $\xi$ takes, the faster the waveform attenuates:

$$
y^{\prime \prime}+2 \xi D^{\alpha} y+y=-\left[400\left(1-e^{-20 x}\right)+100 \sin (6 \pi x)\right] .
$$

Case 5 (see [31]). Considering the following fractionalorder wave equation, in this case, we take the friction force $f=200 e^{-x} \cos (2 \pi x)$. When $m=4$ and $\alpha=0.8$, the numerical results with $\xi=0.01,0.15$, and 0.6 are shown in Figure 12. Figure 12 shows that the vibration displacement solutions are still attenuated waveform, and the 


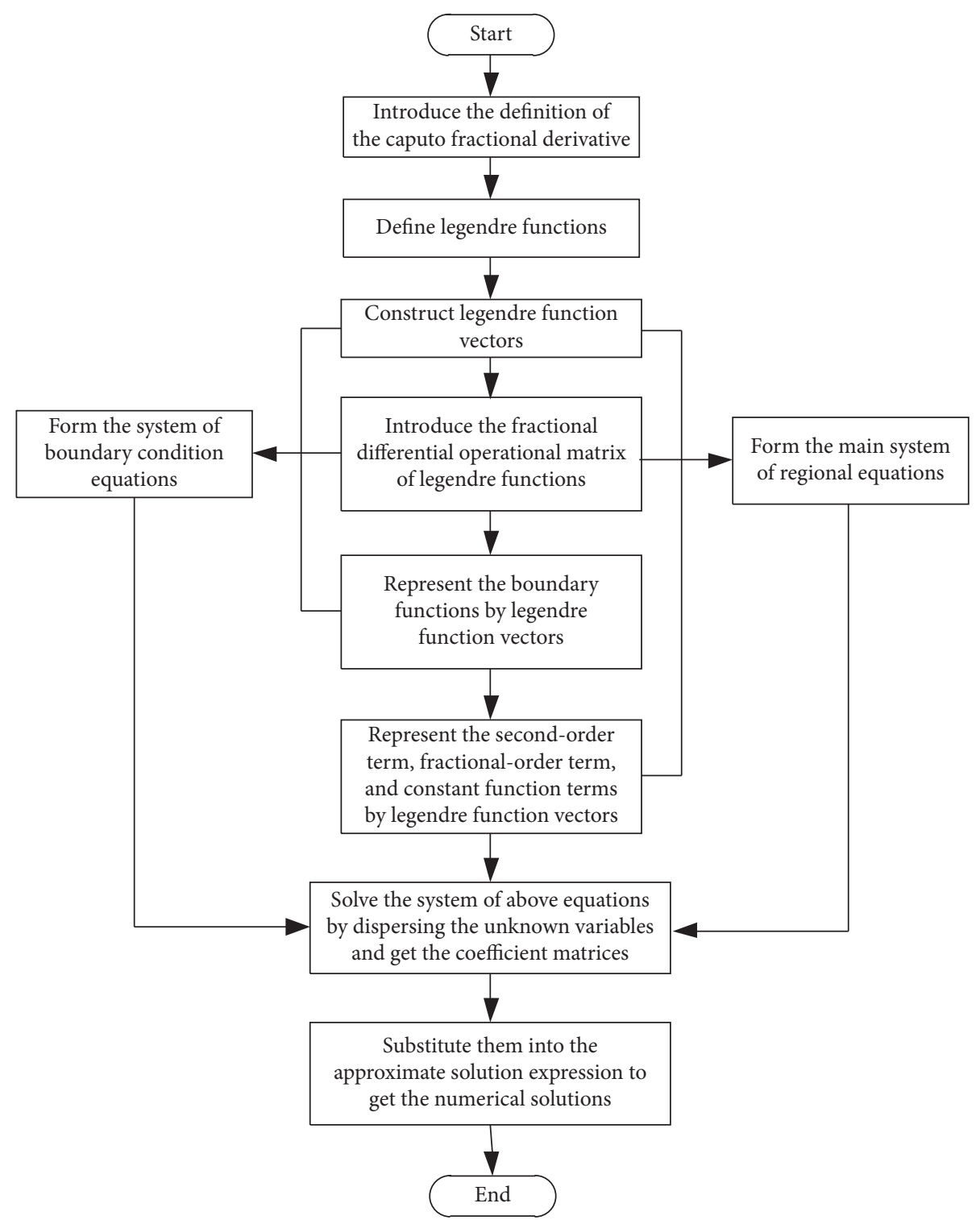

Figure 3: Algorithm flowchart.

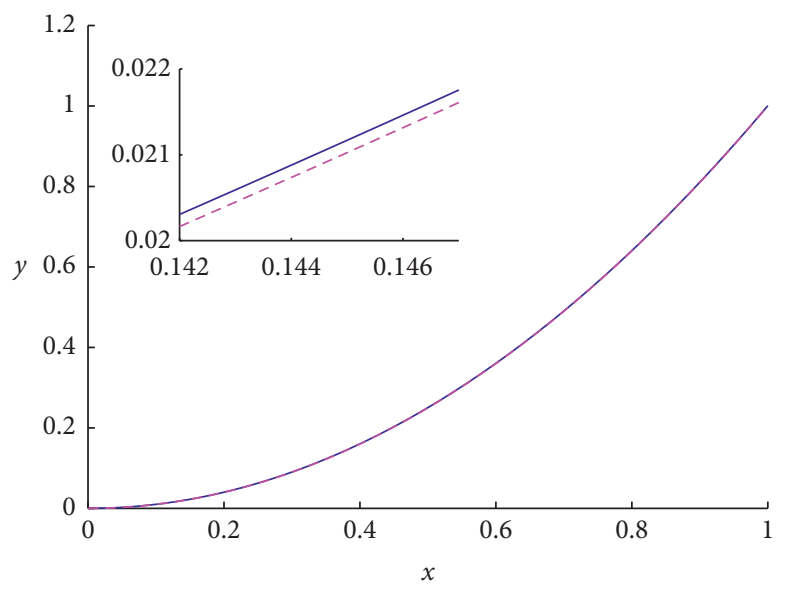

_ Numer. sol.

_. - Anal. sol.

FIgURE 4: Numerical and analytical results with $m=4$. 


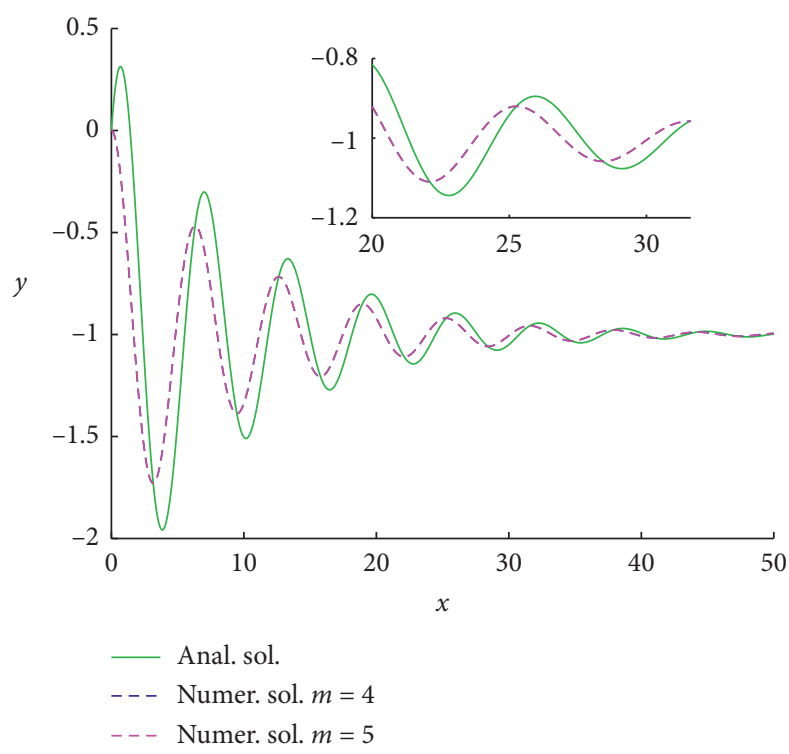

Figure 5: Analytical solutions and numerical vibration displacement solutions with $m=4,5$.

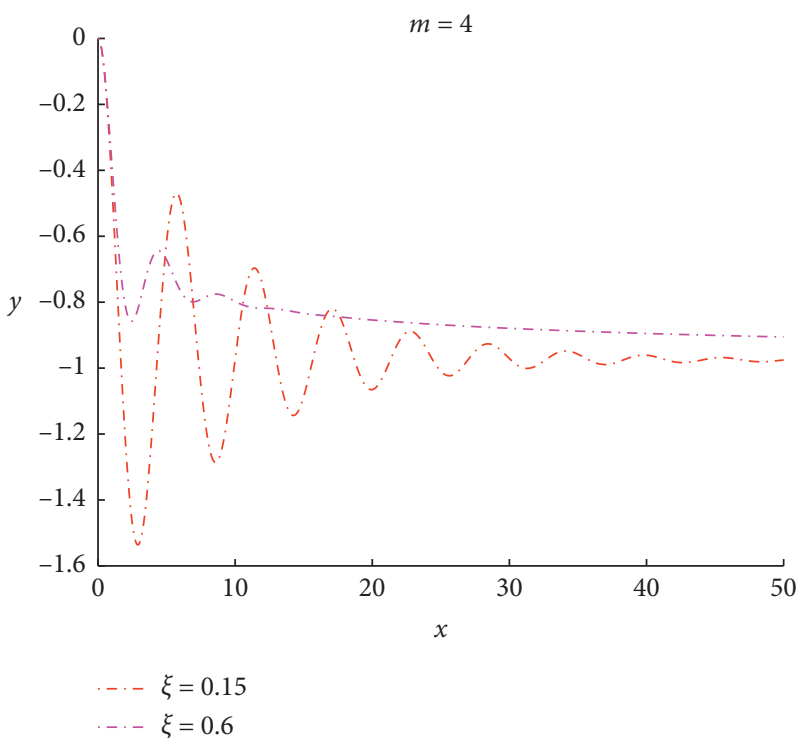

FIgURE 6: Numerical vibration displacement solutions with $\xi=0.15$ and 0.6.

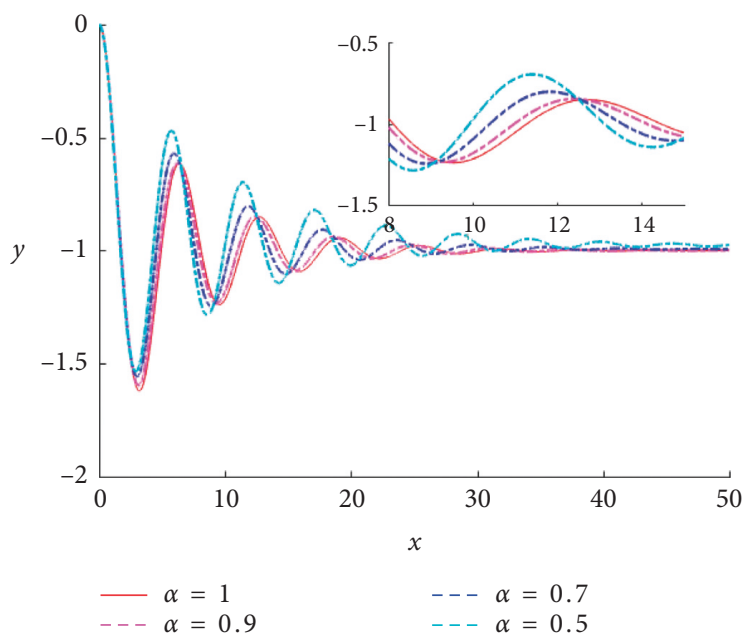

FIGURE 7: Numerical vibration displacement solutions with $\alpha=0.5,0.7,0.9$, and 1 . 


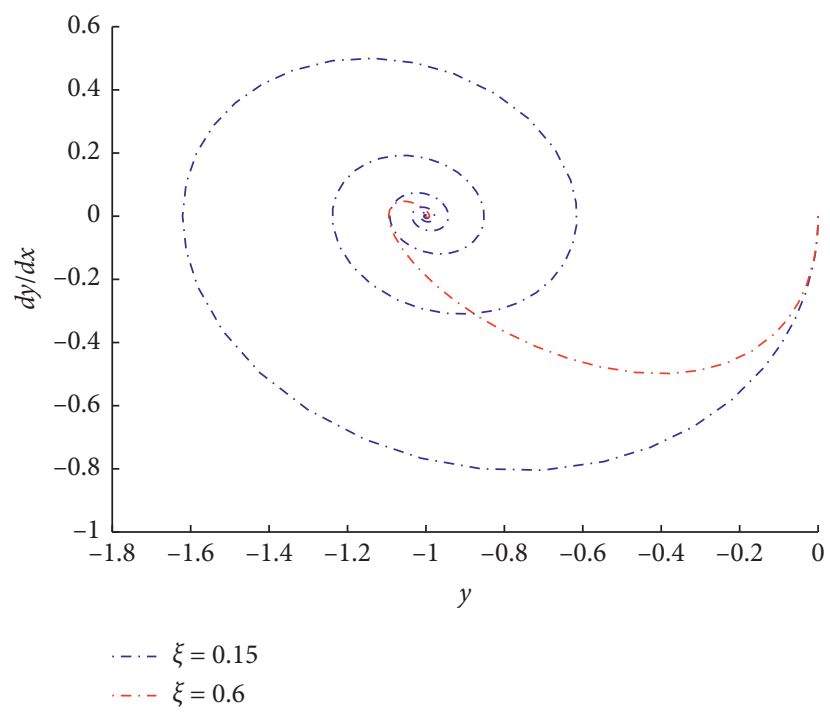

FIgURE 8: The phase diagram with $\xi=0.15$ and $\xi=0.6$.

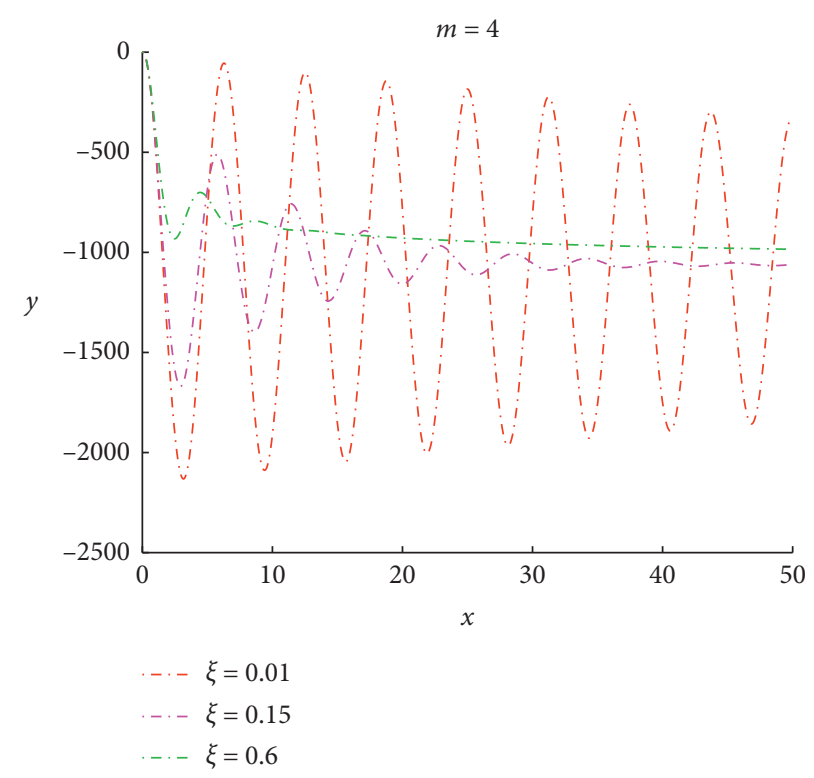

FIGURE 9: Numerical vibration displacement solutions with $\xi=0.01,0.15$, and 0.6.

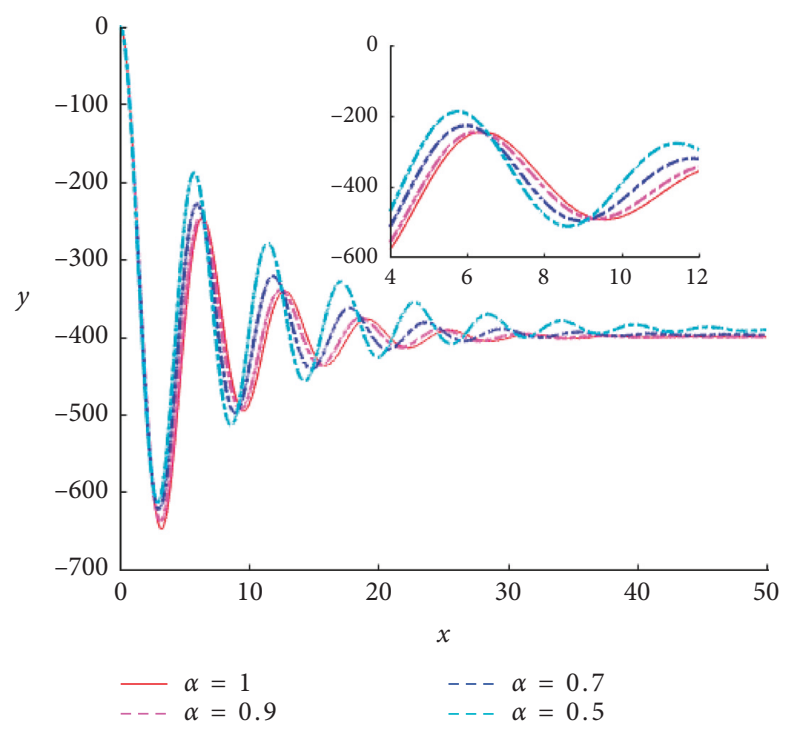

FigURE 10: Numerical vibration displacement solutions with $\alpha=0.5,0.7,0.9$, and 1 . 


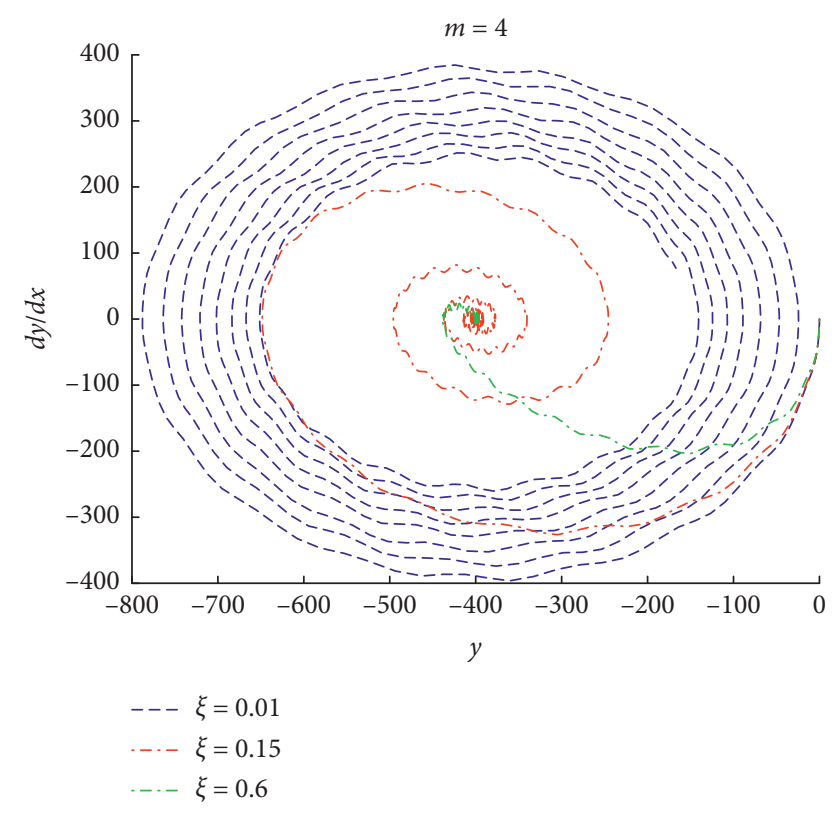

FIGURE 11: The phase diagram with $\xi=0.01, \xi=0.15$, and $\xi=0.6$.

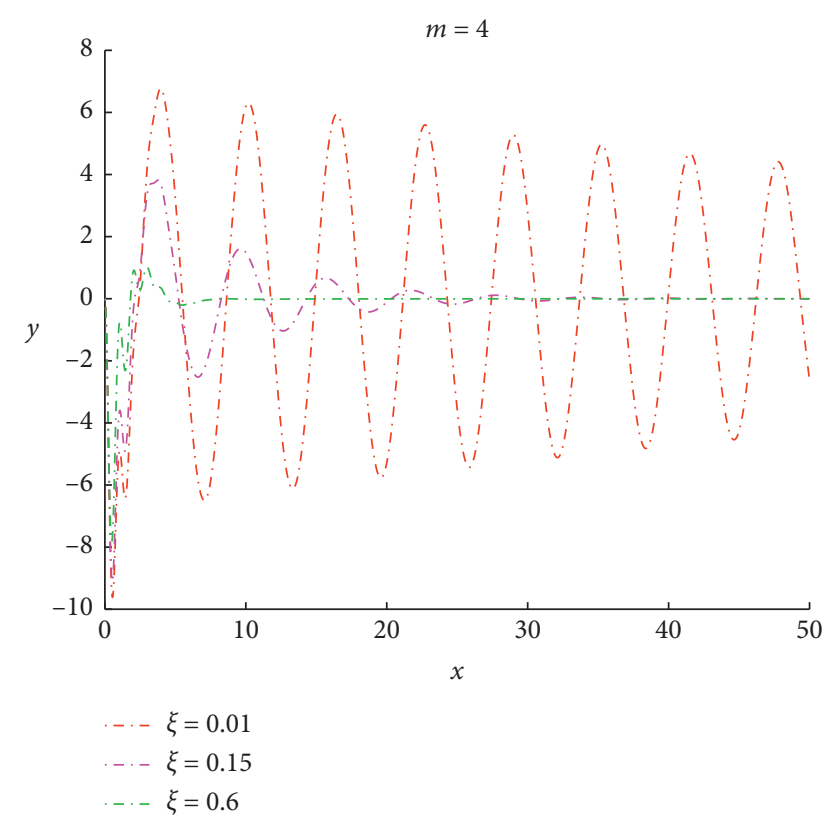

Figure 12: Numerical vibration displacement solutions with $\xi=0.01,0.15$, and 0.6 .

waveform is equiperiodic when $\xi=0.01$. Moreover, the waveforms no longer change regularly with $\xi$ increasing. The numerical results show that there is a large fluctuation at the beginning, and the fluctuation process tends to be smooth with $x$ increasing. When $m=4$ and $\xi=0.15$, the numerical results with $\alpha=0.6,0.7,0.8,0.9$, and 1 are shown in Figure 13. When $m=4$ and $\alpha=1$, the phase diagram with $\xi=0.01, \xi=0.15$, and $\xi=0.6$ is shown in Figure 14.

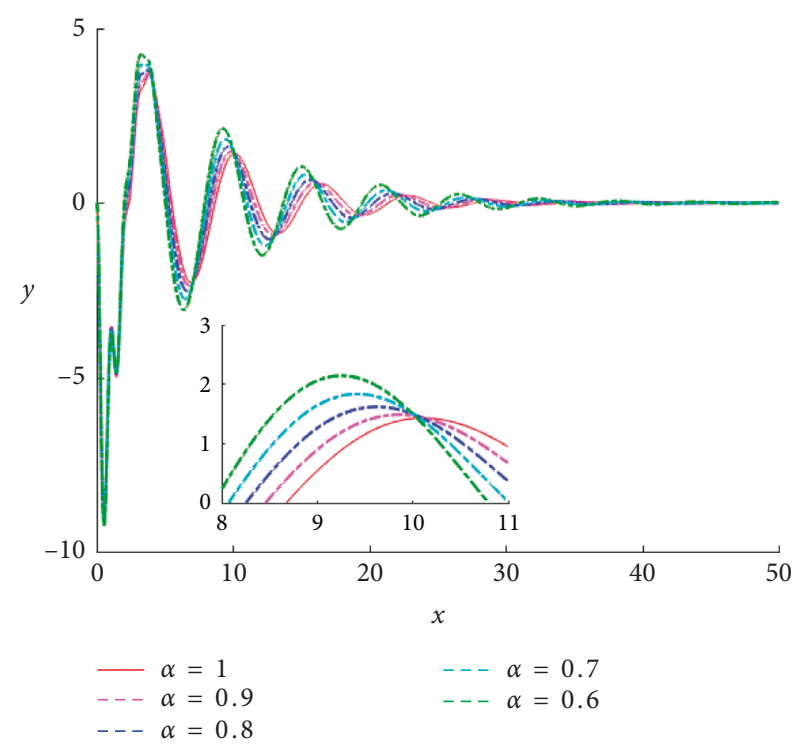

FIgURE 13: Numerical vibration displacement solutions with $\alpha=0.6,0.7,0.8,0.9$, and 1 .

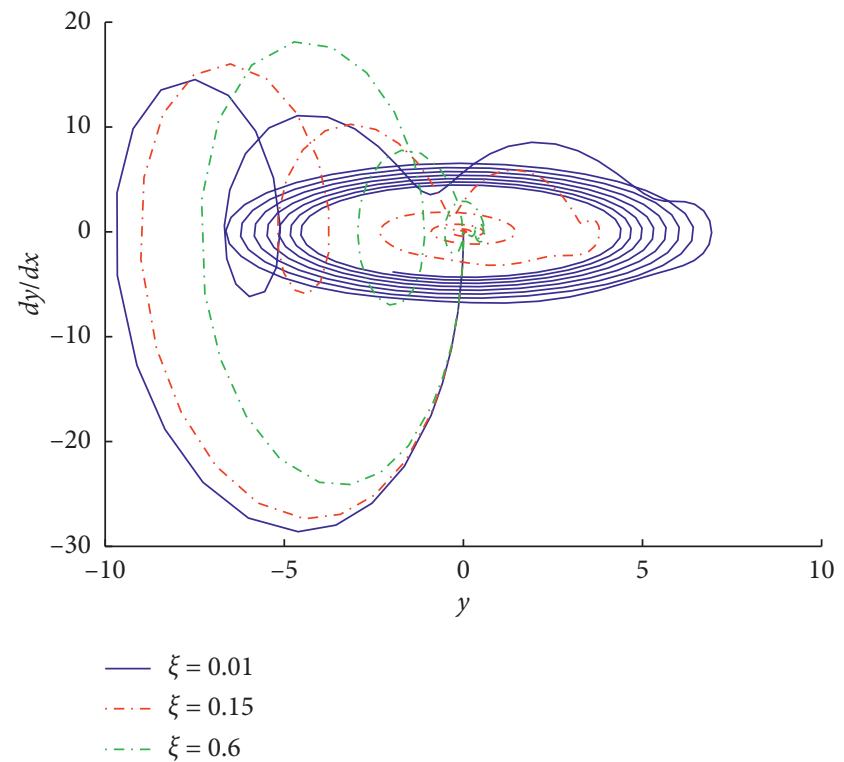

FIGURE 14: The phase diagram with $\xi=0.01, \xi=0.15$, and $\xi=0.6$.

$$
y^{\prime \prime}+2 \xi D^{\alpha} y+y=-200 e^{-x} \cos (2 \pi x)
$$

\section{Conclusions}

In this paper, a fractional differential equation model of drawing self-excited vibration is proposed based on the mechanical system hunting phenomenon. The fractionalorder Legendre function method is utilized to obtain the vibration displacement solutions of the given model, and the established model and proposed algorithm are analyzed from several specific numerical experiments. 


\section{Data Availability}

The data used to support the findings of this study are included within the article.

\section{Conflicts of Interest}

All authors declare that they have no conflicts of interest.

\section{Authors' Contributions}

All authors contributed equally to this manuscript.

\section{Acknowledgments}

This work was supported by National Key R\&D Program of China (2018YFB1307902), major program of National Natural Science Foundation of China (U1710254), Key Research and Development Program of Shanxi Province (201703D111003), Youth Science Foundation of Natural Science Foundation of China (51505417), Youth Science Foundation of Natural Science Foundation of Hebei Province (E2016203294), and Shanxi Province Science and Technology Major Projects (20181102015).

\section{References}

[1] W. J. Ding, Self-Excited Vibration, Tsinghua University Press, Beijing, China, 2009.

[2] P. A. Kolkman, Flow-induced gate vibrations, Ph.D. thesis, Delft University of Technology, Delft, The Netherlands, 1976.

[3] E. Naudascher and D. Rockwell, Flow-Induced Vibrations-An Engineering Guide, Dover Publications, New York, NY, USA, 1994.

[4] C. D. Erdbrink, Modelling flow-induced vibrations of gates in hydraulic structures, Ph.D. thesis, University of Amsterdam, Amsterdam, The Netherlands, 2014.

[5] Q. Wang, H. E. Psillakis, and C. Sun, "Adaptive cooperative control with guaranteed convergence in time-varying networks of nonlinear dynamical systems," IEEE Transactions on Cybernetics, 2019.

[6] Q. Wang, H. E. Psillakis, and C. Sun, "Cooperative control of multiple agents with unknown high-frequency gain signs under unbalanced and switching topologies," IEEE Transactions on Automatic Control, vol. 64, no. 6, pp. 2495-2501, 2018.

[7] H. Huang, X. Chen, B. Zhang, and J. Wang, "High accuracy navigation information estimation for inertial system using the multi-model EKF fusing adams explicit formula applied to underwater gliders," ISA Transactions, vol. 66, pp. 414-424, 2017.

[8] H. Huang, J. Zhou, J. Zhang et al., "Attitude estimation fusing Quasi-Newton and cubature Kalman filtering for inertial navigation system aided with magnetic sensors," IEEE Access, vol. 6, pp. 28755-28767, 2018.

[9] G. Y. Yang, W. W. Li, and J. Y. Shi, "High speed and highquality drawing technology test for PC steel strand," Metal Products, vol. 41, no. 1, pp. 20-24, 2015.

[10] S. Y. Hao, J. Zhang, D. Y. Wen et al., "The mechanism and elimination method of chattering grain of cold drawn steel pipe," Heavy Machinery, no. 6, pp. 25-28, 2001.
[11] N. B. Yang, "Vibration and vibration of rolling mill are analyzed by finite element method," CFHI Technology, no. 1, pp. 139-152, 1994.

[12] X. Y. Lu, Q. Y. Ye, and Z. H. Qu, "Analysis and solution of selfexcited vibration of a kind of rolling mill," Journal of Shanghai University of Science and Technology, vol. 26, no. 2, pp. 141145, 2004.

[13] C. D. Erdbrink and V. V. Krzhizhanovskaya, "Differential evolution for system identification of self-excited vibrations," Journal of Computational Science, vol. 10, pp. 360-369, 2015.

[14] U. J. F. Aarsnes and O. M. Aamo, "Linear stability analysis of self-excited vibrations in drilling using an infinite dimensional model," Journal of Sound and Vibration, vol. 360, pp. 239-259, 2016.

[15] Z. Zhang, S. Oberst, and J. C. S. Lai, "A non-linear friction work formulation for the analysis of self-excited vibrations," Journal of Sound and Vibration, vol. 443, pp. 328-340, 2019.

[16] I. Podlubny, "Geometric and physical interpretation of fractional integration and fractional differentiation," Fractional Calculus and Applied Analysis, vol. 5, no. 4, pp. 367386, 2002.

[17] P. J. Torvik and R. L. Bagley, "On the appearance of the fractional derivative in the behavior of real materials," Journal of Applied Mechanics, vol. 51, no. 2, pp. 294-298, 1984.

[18] J. Liang, Y. Chen, and R. Fullmer, "Simulation studies on the boundary stabilization and disturbance rejection for fractional diffusion-wave equation," in Proceedings of the 2004 American Control Conference, vol. 38, pp. 339-354, Boston, MA, USA, July 2004.

[19] M. H. Farahi, J. E. Rubio, and D. A. Wilson, "The optimal control of the linear wave equation," International Journal of Control, vol. 63, no. 5, pp. 833-848, 1996.

[20] F. D. Araruna, E. Fernández-Cara, and L. C. D. Silva, "Hierarchic control for the wave equation," Journal of Optimization Theory and Applications, vol. 178, no. 1, pp. 264-288, 2018.

[21] J. Jiang, J. L. G. Guirao, H. Chen, and D. Cao, “The boundary control strategy for a fractional wave equation with external disturbances," Chaos, Solitons \& Fractals, vol. 121, pp. 92-97, 2019.

[22] H. Chen, S. Lü, and W. Chen, "Spectral methods for the time fractional diffusion-wave equation in a semi-infinite channel," Computers \& Mathematics with Applications, vol. 71, no. 9, pp. 1818-1830, 2016.

[23] J. Chen, F. Liu, V. Anh, S. Shen, Q. Liu, and C. Liao, "The analytical solution and numerical solution of the fractional diffusion-wave equation with damping," Applied Mathematics and Computation, vol. 219, no. 4, pp. 1737-1748, 2012.

[24] W. J. Ding, Self-excited Vibration in Engineering, Jilin Education Press, Jilin, China, 1988.

[25] J. Y. Wan, Analysis and Systematic Study of Drawing Process Parameters of Steel Strand, 2015.

[26] J. Xie and M. Yi, "Numerical research of nonlinear system of fractional Volterra-Fredholm integral-differential equations via Block-Pulse functions and error analysis," Journal of Computational and Applied Mathematics, vol. 345, pp. 159167, 2019.

[27] J. Xie, Z. Yao, H. Gui, F. Zhao, and D. Li, “A two-dimensional Chebyshev wavelets approach for solving the Fokker-Planck equations of time and space fractional derivatives type with variable coefficients," Applied Mathematics and Computation, vol. 332, pp. 197-208, 2018.

[28] F. Mohammadi and C. Cattani, "A generalized fractionalorder Legendre wavelet Tau method for solving fractional 
differential equations," Journal of Computational and Applied Mathematics, vol. 339, pp. 306-316, 2018.

[29] Y. Chen, Y. Sun, and L. Liu, "Numerical solution of fractional partial differential equations with variable coefficients using generalized fractional-order Legendre functions," Applied Mathematics and Computation, vol. 244, pp. 847-858, 2014.

[30] S. Kazem, S. Abbasbandy, and S. Kumar, "Fractional-order Legendre functions for solving fractional-order differential equations," Applied Mathematical Modelling, vol. 37, no. 7, pp. 5498-5510, 2013.

[31] F. Peng, L. Wang, and J. Xiao, "Dynamic load identification for mass time-varying systems," Journal of Hunan University (Natural Science Edition), vol. 43, no. 8, pp. 52-56, 2016. 


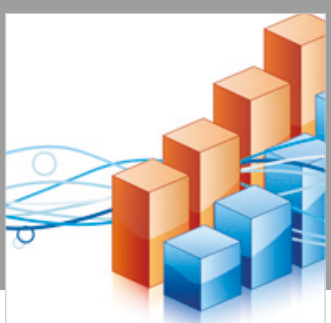

Advances in

Operations Research

\section{-n-m}
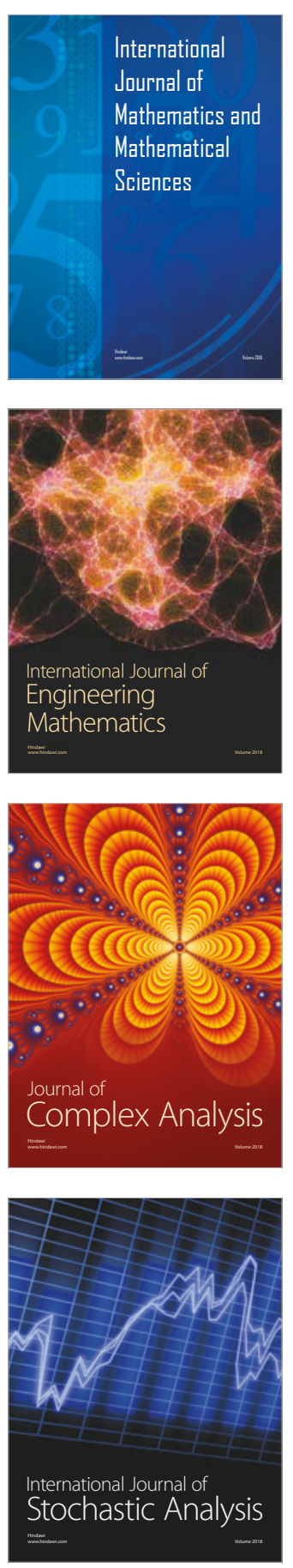
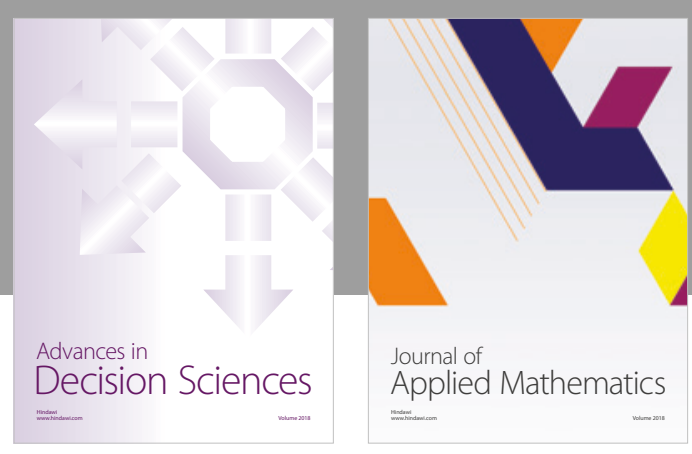

Journal of

Applied Mathematics
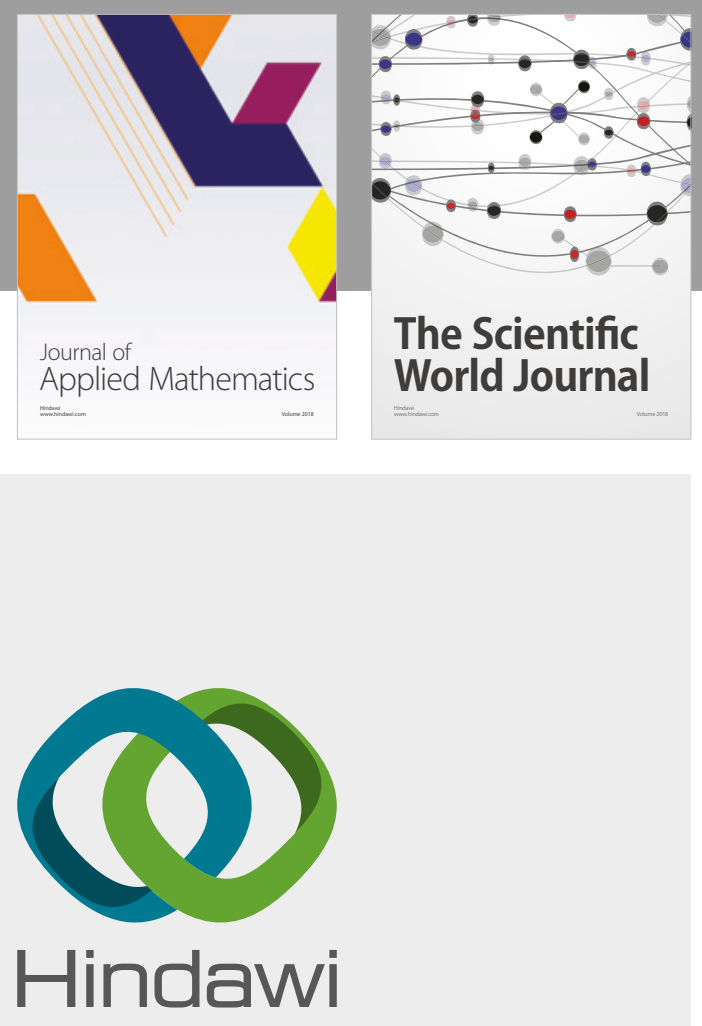

Submit your manuscripts at

www.hindawi.com

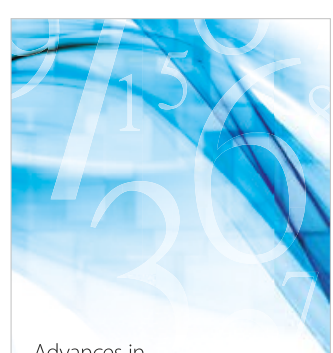

Advances in
Numerical Analysis
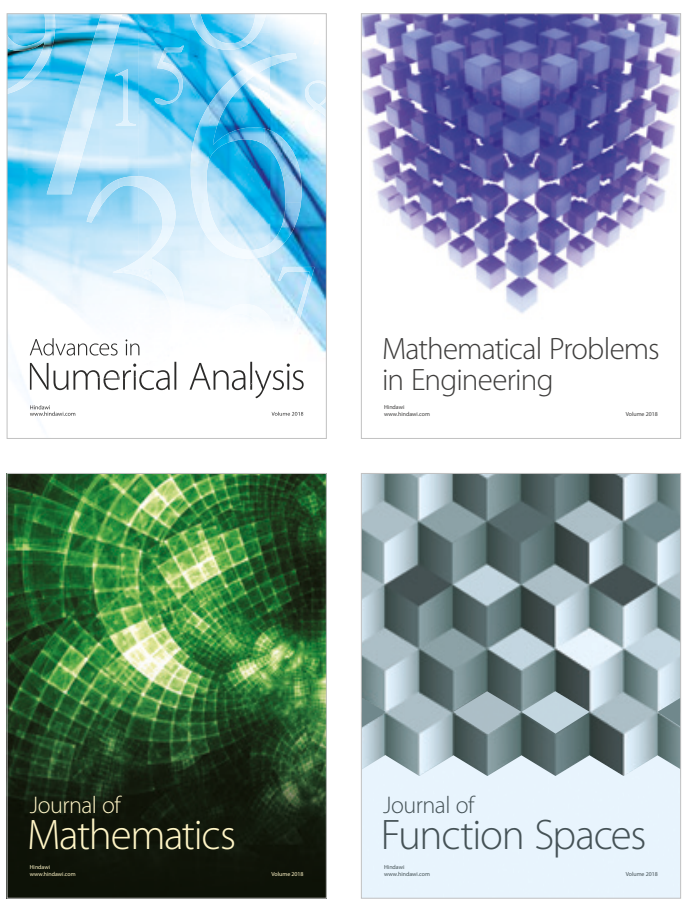

Mathematical Problems in Engineering

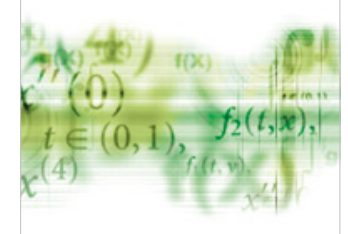

International Journal of

Differential Equations

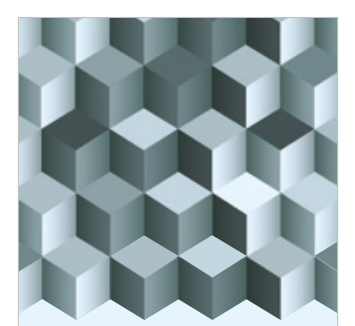

Journal of

Function Spaces
The Scientific

World Journal

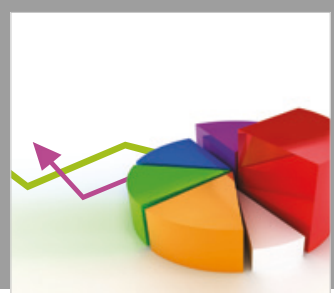

Journal of

Probability and Statistics
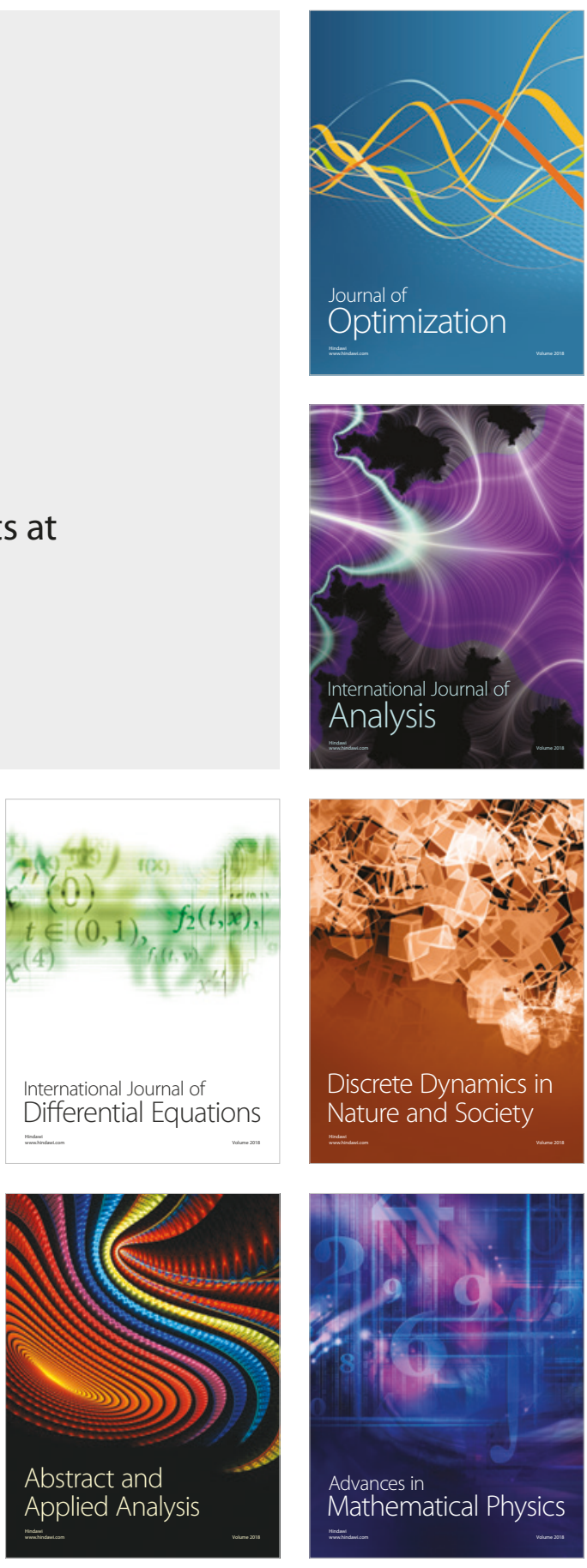